\title{
Condições higiênico-sanitárias de unidades de alimentação e nutrição de escolas
}

\author{
Hygienic-sanitary conditions of school food and nutrition units \\ Condiciones higiénico-sanitarias de las unidades de alimentación y nutrición escolar
}

Recebido: 02/08/2021 | Revisado: 10/08/2021 | Aceito: 15/08/2021 | Publicado: 1/08/2021

Gilvânia da Conceição Rocha

ORCID: https://orcid.org/0000-0002-8493-6054 Centro Universitário de Saúde, Ciências Humanas e Tecnológicas do Piaú, Brasil E-mail: gilvaniacrocha@gmail.com

Maria de Fátima Sousa Barros Vilarinho

ORCID: https://orcid.org/0000-0003-3226-1160

Universidade Federal do Piauí, Brasil

E-mail: nutrifatimabarros@gmail.com

Aline Tavares Gomes
ORCID: https://orcid.org/0000-0001-6964-6748

Universidade Federal do Piauí, Brasil

E-mail: alinettavaresg@gmail.com

Vanessa Maria Oliveira Viana

ORCID: https://orcid.org/0000-0002-9876-6156 Universidade Federal do Piauí, Brasil E-mail: vanessamariaok@gmail.com

Dinah Alencar Melo Araújo

ORCID: https://orcid.org/0000-0003-0922-349X Universidade Federal do Piauí, Brasil E-mail: jcamo@hotmail.com

Izabela Arrais Vieira

ORCID: https://orcid.org/0000-0002-8199-8428 Centro de Ensino Unificado de Teresina, Brasil E-mail: izabela.arrais@ hotmail.com

Dennisy Kelle Gonçalves de Melo Bezerra ORCID: https://orcid.org/0000-0002-1507-7591 Centro Universitário Santo Agostinho, Piauí, Brasil E-mail: kellebezerranutri@gmail.com Luis Paulo Teixeira da Silva ORCID: https://orcid.org/0000-0002-9080-7195 Universidade Federal do Piauí, Brasil E-mail: Ipteixeirasilva@gmail.com

Taciana Tavares de Sousa ORCID: https://orcid.org/0000-0003-2139-8348 Universidade Norte do Paraná, Brasil E-mail: tacytavares03@gmail.com Maria Gessica Ozório Brito ORCID: https://orcid.org/0000-0002-4263-1033 Centro de Ensino Unificado de Teresina, Brasil E-mail: gessikabritto@ hotmail.com

Ádilla Michele da Silva dos Santos ORCID: https://orcid.org/0000-0003-3673-5239 Centro de Ensino Unificado de Teresina, Brasil E-mail: adillamichele@outlook.com

Clicia Oliveira Sales

ORCID: https://orcid.org/0000-0002-5771-6822 Centro de Ensino Unificado de Teresina, Brasil E-mail: cliciasales@outlook.com

\section{Resumo}

As Unidades de Alimentação e Nutrição devem contribuir para manter, melhorar ou recuperar a saúde da clientela atendida. Contudo, más condições de higiene nesses locais podem contribuir para a ocorrência de doenças transmitidas por alimentos. Este estudo tem como objetivo discutir os resultados de pesquisas acerca das condições higiênico-sanitárias de Unidades de Alimentação e Nutrição de escolas brasileiras. Trata-se de revisão integrativa de artigos publicados entre 2017 e 2021, e indexados nas bases de dados LILACS, SciELO e Google Scholar. Utilizou-se os termos: "boas práticas de fabricação", "alimentação escolar", "produção de alimentos" e "controle de qualidade" e suas versões em inglês. Esta revisão baseou-se em onze estudos, os quais utilizaram a lista de verificação de Boas Práticas para avaliarem e classificarem o risco sanitário das unidades analisadas. A maioria dos estudos observou que 
as Unidades de Alimentação e Nutrição de escolas de diferentes regiões do Brasil apresentaram condições higiênicosanitárias parcialmente adequadas, segundo classificação da Resolução da Diretoria Colegiada $n^{\circ}$ 275/2002 da Agência Nacional de Vigilância Sanitária. As inconformidades dos locais ocorreram em todos os blocos avaliados pelos estudos, sendo mais frequentes nos edifícios e instalações. Verifica-se a necessidade de maior atenção dos órgãos de controle sanitário para com as Unidades de Alimentação e Nutrição de escolas, considerando que a alimentação ofertada nesse ambiente deve ser de qualidade sanitária e nutricional, contribuindo para a saúde e rendimento acadêmico dos estudantes.

Palavras-chave: Boas práticas de fabricação; Alimentação escolar; Produção de alimentos; Controle de qualidade.

\begin{abstract}
The Food and Nutrition Units must contribute to maintaining, improving or restoring the health of the clientele served. However, poor hygiene conditions in these places can contribute to the occurrence of foodborne illnesses. This study aims to discuss the results of research on the hygienic-sanitary conditions of Food and Nutrition Units in Brazilian schools. This is an integrative review of articles published between 2017 and 2021, and indexed in LILACS, SciELO and Google Scholar databases. The terms: "good manufacturing practices", "school feeding", "food production" and "quality control" and their English versions were used. This review was based on eleven studies, which used the Good Practices checklist to assess and classify the health risk of the analyzed units. Most studies observed that the Food and Nutrition Units of schools in different regions of Brazil had partially adequate hygienic-sanitary conditions, according to the classification of the Resolution of the Collegiate Board No. 275/2002 of the National Health Surveillance Agency. Nonconformities in the locations occurred in all blocks assessed by the studies, being more frequent in buildings and facilities. There is a need for greater attention from health control agencies to the Food and Nutrition Units of schools, considering that the food offered in this environment must be of sanitary and nutritional quality, contributing to the health and academic performance of students.
\end{abstract}

Keywords: Good manufacturing practices; School meals; Food production; Quality control.

\title{
Resumen
}

Las Unidades de Alimentación y Nutrición deben contribuir a mantener, mejorar o restaurar la salud de la clientela atendida. Sin embargo, las malas condiciones de higiene en estos lugares pueden contribuir a la aparición de enfermedades transmitidas por los alimentos. Este estudio tiene como objetivo discutir los resultados de la investigación sobre las condiciones higiénico-sanitarias de las Unidades de Alimentación y Nutrición en las escuelas brasileñas. Se trata de una revisión integradora de artículos publicados entre 2017 y 2021, e indexados en las bases de datos LILACS, SciELO y Google Scholar. Se utilizaron los términos: "buenas prácticas de fabricación", "alimentación escolar", "producción de alimentos" y "control de calidad” y sus versiones en inglés. Esta revisión se basó en once estudios, que utilizaron el checklist de Buenas Prácticas para evaluar y clasificar el riesgo para la salud de las unidades analizadas. La mayoría de los estudios observaron que las Unidades de Alimentación y Nutrición de las escuelas en diferentes regiones de Brasil tenían condiciones higiénico-sanitarias parcialmente adecuadas, según la clasificación de la Resolución del Consejo Colegiado No. 275/2002 de la Agencia Nacional de Vigilancia Sanitaria. Las no conformidades en las ubicaciones ocurrieron en todos los bloques evaluados por los estudios, siendo más frecuentes en edificios e instalaciones. Existe la necesidad de una mayor atención por parte de los organismos de control de la salud a las Unidades de Alimentación y Nutrición de las escuelas, considerando que los alimentos que se ofrecen en este entorno deben ser de calidad sanitaria y nutricional, contribuyendo a la salud y el rendimiento académico de los estudiantes.

Palabras clave: Buenas prácticas de fabricación; Comidas del colegio; La producción de alimentos; Control de calidad.

\section{Introdução}

As Unidades de Alimentação e Nutrição (UAN) consistem em estabelecimentos que realizam o preparo e distribuição de alimentos para coletividades sadia ou enferma, de modo organizado, utilizando cardápio balanceado e de acordo com padrões dietéticos e higiênico-sanitários estabelecidos (Martins \& Spinelli, 2020). Essas unidades têm como objetivo contribuir para manutenção, melhora ou recuperação da saúde da clientela atendida, atendendo suas necessidades nutricionais (Conselho Federal de Nutricionistas, 2018).

A promoção da saúde está relacionada com a segurança alimentar, uma vez que a oferta de uma alimentação segura associada a hábitos alimentares saudáveis reduz os riscos para doenças crônicas não transmissíveis, contribuindo para a qualidade de vida das pessoas. As Unidades de Alimentação e Nutrição quando bem estruturadas e focadas na segurança 
alimentar podem ser consideradas como fator de promoção de saúde e de desenvolvimento socioeconômico (Oliveira, Oliveira, Mallet \& Souza, 2020).

Para a obtenção de alimentos seguros, do ponto de vista sanitário, é necessário que haja o controle de qualidade de todo o processo de produção do alimento (Ferreira, Lima \& Aguiar, 2020). As unidades de produção de alimentos devem implantar algumas regras básicas para manusear de modo higiênico os alimentos, denominadas de boas práticas, que visam controlar, prevenir, eliminar ou reduzir a contaminação biológica, física ou química, desde a matéria-prima até o produto final, assegurando a oferta de alimentos seguros para o consumo e a saúde do consumidor (Silva et al., 2016). Todavia, condições higiênico-sanitárias precárias, ausência de capacitação dos trabalhadores que atuam na manipulação dos alimentos e a falta de fiscalização pelas autoridades sanitárias nesses locais são fatores que contribuem de modo importante para a ocorrência de Doenças Transmitidas por Alimentos (DTA) (Sousa, Paz, Carvalho, Bezerra \& Landim, 2020).

O consumo de alimentos fora de casa pode representar riscos à saúde caso os estabelecimentos produtores desses alimentos não adotem as condutas preconizadas em normativas e legislações sanitárias. O não seguimento e/ou não adoção das boas práticas de manipulação expõe os alimentos aos perigos de contaminação, estando o consumo destes associado ao aumento do risco de DTA (Ferrari \& Fonseca, 2019).

As DTA são preocupações relacionadas à saúde pública que se tornam mais relevantes no âmbito de Unidades de Alimentação e Nutrição de escolas (Silva et al., 2016), uma vez que o público atendido nessas unidades, normalmente, são crianças, grupo no qual essas doenças tendem a ser mais graves. Contudo, estudos relatam a ocorrência de falhas relevantes nessas unidades como comprometimento da infraestrutura, precariedade nos aspectos higiênico sanitários das instalações, equipamentos e utensílios, e inadequações no comportamento dos manipuladores, o que representa um risco em potencial para contaminação dos alimentos e ocorrência de DTA (Freitas, Cunha, Machado, Silva \& Raghiante, 2017).

Considerando que más condições higiênico-sanitárias em estabelecimentos produtores de alimentos podem representar um risco à saúde do público atendido, esse estudo teve como objetivo discutir os resultados de pesquisas realizadas no Brasil acerca das condições higiênico-sanitárias de Unidades de Alimentação e Nutrição de escolas.

\section{Metodologia}

Trata-se de uma revisão de literatura integrativa, cujo levantamento dos artigos científicos foi realizado nas bases de dados LILACS (Literatura Latino-Americana e do Caribe em Ciências da Saúde), SciELO (Scientific Eletronic Library Online) e Google Scholar, a partir dos seguintes termos e suas combinações em português: "boas práticas de fabricação", "alimentação escolar", "produção de alimentos" e "controle de qualidade", e em inglês: "good manufacturing practices", "school feeding", "food production" e "quality control".

Foram incluídos artigos científicos originais disponíveis no formato completo, publicados em periódicos indexados entre os anos de 2017 e 2021 nos idiomas português e inglês que avaliaram as condições sanitárias e boas práticas de higiene e manipulação em Unidades de Alimentação e Nutrição de escolas localizadas em diferentes regiões brasileiras. Adotou-se como critérios de exclusão: artigos de revisão, teses e dissertações, cartas ao editor, editoriais, boletins epidemiológicos, estudos duplicados, pesquisas realizadas em outros países ou que não avaliaram as condições higiênico-sanitárias de Unidades de Alimentação e Nutrição de escolas com base na legislação sanitária vigente no Brasil.

Os estudos selecionados avaliaram as condições higiênico-sanitárias das Unidades de Alimentação e Nutrição através de lista de verificação de Boas Práticas, sendo que a maioria utilizou a versão específica para escolas validada por Stedefeldt, Cunha, Silva Júnior, Silva e Oliveira (2013) e os demais utilizaram versão adaptada da Resolução da Diretoria Colegiada (RDC) $n^{\circ}$ 275/2002 da Agência Nacional de Vigilância Sanitária (Anvisa) (Anvisa, 2002). De modo geral, a lista de 
verificação era composta dos seguintes blocos de avaliação: edifícios e instalações da área de preparo de alimentos; equipamentos para temperatura controlada; manipuladores; recebimento; processos e procedimentos; e higienização ambiental.

Com base na pontuação geral obtida na lista de verificação, isto é, somando-se as pontuações de cada bloco, as Unidades de Alimentação e Nutrição foram classificadas quanto ao risco sanitário, sendo: "muito alto risco (0-25\% de conformidade dos itens)", "alto risco (26-50\% de conformidades dos itens)", "risco regular (51-75\% de conformidades dos itens)", "baixo risco (76-90\% de conformidades dos itens)" e "muito baixo risco (91-100\% de conformidades dos itens)" segundo classificação do instrumento validado por Stedefeldt et al (2013); e em: "inadequado ( $<50 \%$ de conformidades dos itens)", "parcialmente adequado (51-75\% de conformidades dos itens)" e "adequado (76-100\% de conformidades dos itens)", classificação estabelecida pela RDC nº 275/2002 da Anvisa (Anvisa, 2002).

Os artigos selecionados foram inicialmente submetidos à leitura dos títulos e resumos e posteriormente lidos na integra para extração dos dados apresentados nessa revisão. A Figura 1 apresenta o fluxograma das etapas do processo de busca e seleção dos estudos.

Figura 1. Fluxograma de busca, seleção e distribuição dos artigos selecionados para a revisão (2017 -2021).

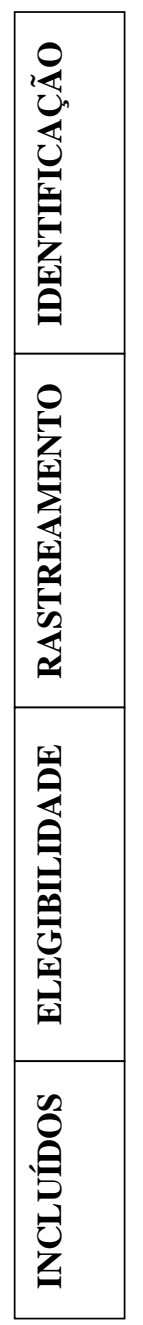

100 artigos encontrados a partir das palavras chaves nas bases de dados

Daqueles, 20 artigos foram excluidos (publicação anterior a 2017), permanecendo 80 estudos

Daqueles, 54 artigos foram excluidos (revisões, teses, trabalhos não disponiveis, fuga do tema, etc.)

Dos 26 artigos, 15 foram excluidos por serem duplicados ou não atenderem aos critérios de inclusão.

11 periódicos foram incluidos. 


\section{Resultados}

Os resultados desta revisão estão apresentados no Quadro 1, no qual estão sintetizados os dados extraídos dos estudos selecionados, quais sejam: autor e ano da pesquisa, objetivo do estudo, metodologia adotada e os principais resultados observados pelos autores quanto às condições de higiene e boas práticas de manipulação de alimentos nas Unidades de Alimentação e Nutrição avaliadas.

Quadro 1. Condições higiênico-sanitárias de Unidades de Alimentação e Nutrição de escolas brasileiras, 2017 a 2021.

\begin{tabular}{|c|c|c|c|}
\hline Autor/ano & Objetivo do estudo & Metodologia & Resultados \\
\hline $\begin{array}{l}\text { Batista e } \\
\text { Vergara } \\
(2017)\end{array}$ & $\begin{array}{l}\text { Averiguar as boas práticas e risco } \\
\text { sanitário em Unidades de } \\
\text { Alimentação e Nutrição de } \\
\text { escolas municipais de } \\
\text { Maracanaú-CE. }\end{array}$ & $\begin{array}{l}\text { Aplicação de lista de } \\
\text { verificação de boas práticas } \\
\text { para Unidades de Alimentação } \\
\text { e Nutrição de escolas, proposta } \\
\text { e validada por Stedefeldt et al } \\
\text { (2013), em } 101 \text { escolas. }\end{array}$ & $\begin{array}{l}83,2 \% \text { das unidades tinham risco sanitário regular } \\
\text { e } 16,8 \% \text { risco sanitário alto, com média de } 55,3 \% \\
\text { de conformidade, representando risco sanitário } \\
\text { regular. As inconformidades estiveram rela- } \\
\text { cionadas especialmente a estrutura física, equi- } \\
\text { pamentos em estado de conservação ruim ou sua } \\
\text { ausência e falhas na higiene pessoal, dos utensílios } \\
\text { e alimentos. }\end{array}$ \\
\hline $\begin{array}{l}\text { Fortes, Brasil, } \\
\text { Silva, Pontes e } \\
\text { Graupe (2017) }\end{array}$ & $\begin{array}{l}\text { Diagnosticar as condições } \\
\text { higiênico-sanitárias das Unidades } \\
\text { de Alimentação e Nutrição de } \\
\text { escolas de educação infantil de } \\
\text { Palmeira das Missões-RS. }\end{array}$ & $\begin{array}{l}\text { Aplicação de lista de } \\
\text { verificação de boas práticas } \\
\text { para Unidades de Alimentação } \\
\text { e Nutrição de escolas, proposta } \\
\text { e validada por Stedefeldt } \text { et al } \\
\text { (2013), em sete escolas. }\end{array}$ & $\begin{array}{l}\text { As unidades apresentaram } 31,5 \% \text { de conformidade, } \\
\text { representando risco sanitário alto. As maiores } \\
\text { inconformidades foram observadas nos blocos } \\
\text { recebimento e equipamentos para temperatura } \\
\text { controlada. }\end{array}$ \\
\hline $\begin{array}{l}\text { Ferro, Fialho, } \\
\text { Pires, Teles e } \\
\text { Santos (2018) }\end{array}$ & $\begin{array}{l}\text { Avaliar condições higiênico- } \\
\text { sanitárias das Unidades de } \\
\text { Alimentação e Nutrição de } \\
\text { escolas públicas do estado do } \\
\text { Tocantins. }\end{array}$ & $\begin{array}{l}\text { Aplicação de lista de } \\
\text { verificação de boas práticas } \\
\text { para Unidades de Alimentação } \\
\text { e Nutrição de escolas, proposta } \\
\text { e validada por Stedefeldt et al } \\
\text { (2013), em } 35 \text { escolas. }\end{array}$ & $\begin{array}{l}\text { As unidades apresentaram } 52,8 \% \text { de conformidade, } \\
\text { portanto com risco sanitário regular. As } \\
\text { inconformidades estiveram mais presentes em } \\
\text { aspectos relativos à infraestrutura e aos } \\
\text { manipuladores. }\end{array}$ \\
\hline $\begin{array}{l}\text { Ribeiro et al. } \\
(2018)\end{array}$ & $\begin{array}{l}\text { Analisar através de check list as } \\
\text { boas práticas realizadas na } \\
\text { manipulação de alimentos em } \\
\text { Unidades de Alimentação e } \\
\text { Nutrição de escolas em um } \\
\text { município localizado no Vale do } \\
\text { Ribeira-SP. }\end{array}$ & $\begin{array}{l}\text { Aplicação de lista de } \\
\text { verificação de boas práticas } \\
\text { para Unidades de Alimentação } \\
\text { e Nutrição de escolas, proposta } \\
\text { e validada por Stedefeldt et al } \\
\text { (2013), em seis escolas. }\end{array}$ & $\begin{array}{l}50 \% \text { das unidades apresentaram risco sanitário alto } \\
\text { e } 50 \% \text { risco sanitário regular, com média geral de } \\
48,5 \% \text { de conformidade, representando risco } \\
\text { sanitário alto. As inconformidades estiveram } \\
\text { relacionadas principalmente aos manipuladores e à } \\
\text { infraestrutura. }\end{array}$ \\
\hline $\begin{array}{l}\text { Rudakoff, } \\
\text { Mouchreck, } \\
\text { Frota e Bastos } \\
\text { (2018) }\end{array}$ & $\begin{array}{l}\text { Avaliar as condições higiênico- } \\
\text { sanitárias das Unidades de } \\
\text { Alimentação e Nutrição de } \\
\text { escolas da rede municipal de } \\
\text { educação de São Luís-MA, e } \\
\text { analisar a qualidade } \\
\text { microbiológica da alimentação } \\
\text { servida e da água dos bebedouros } \\
\text { dessas escolas. }\end{array}$ & $\begin{array}{l}\text { Aplicação de lista de } \\
\text { verificação de boas práticas } \\
\text { para Unidades de Alimentação } \\
\text { e Nutrição de escolas, proposta } \\
\text { e validada por Stedefeldt et al } \\
\text { (2013), em } 30 \text { escolas. }\end{array}$ & $\begin{array}{l}\text { Todas as unidades apresentaram risco sanitário } \\
\text { regular, com } 69,7 \% \text { de conformidade. Os pontos } \\
\text { mais críticos ocorreram nos blocos processos e } \\
\text { procedimentos e equipamentos para temperatura } \\
\text { controlada. }\end{array}$ \\
\hline $\begin{array}{l}\text { Soares et al. } \\
\text { (2018) }\end{array}$ & $\begin{array}{l}\text { Analisar as condições higiênico- } \\
\text { sanitárias de Unidades de } \\
\text { Alimentação e Nutrição de } \\
\text { escolas de um município do } \\
\text { estado do Rio de Janeiro. }\end{array}$ & $\begin{array}{l}\text { Aplicação de lista de } \\
\text { verificação de boas práticas } \\
\text { para Unidades de Alimentação } \\
\text { e Nutrição de escolas, proposta } \\
\text { e validada por Stedefeldt } \text { et al } \\
\text { (2013), em nove escolas. }\end{array}$ & $\begin{array}{l}\text { As unidades apresentaram risco sanitário regular, } \\
\text { com } 64,5 \% \text { de conformidade. As inconformidades } \\
\text { estiveram relacionadas principalmente a } \\
\text { higienização ambiental e processos e } \\
\text { procedimentos. }\end{array}$ \\
\hline
\end{tabular}




\begin{tabular}{|c|c|c|c|}
\hline $\begin{array}{l}\text { Mendes, } \\
\text { Monteiro, } \\
\text { Carvalho e } \\
\text { Bezerra } \\
(2019)\end{array}$ & $\begin{array}{l}\text { Avaliar as estruturas físicas e as } \\
\text { condições higiênico-sanitárias } \\
\text { quanto à produção de refeições } \\
\text { em Unidades de Alimentação e } \\
\text { Nutrição de escolas em Teresina- } \\
\text { PI. }\end{array}$ & $\begin{array}{l}\text { Aplicação de lista de } \\
\text { verificação de boas práticas } \\
\text { para Unidades de Alimentação } \\
\text { e Nutrição, adaptada da RDC } \\
275 / 2002 \text {, em duas escolas de } \\
\text { tempo integral do município. }\end{array}$ & $\begin{array}{l}\text { As unidades apresentaram 83,5\% de conformidade, } \\
\text { considerado "adequado" pela RDC } 275 / 2002 \text {. As } \\
\text { não conformidades estiveram relacionadas ao } \\
\text { desconhecimento ou ausência de Procedimentos } \\
\text { Operacionais Padronizados (POP) e Manual de } \\
\text { Boas Práticas (MBP) e a não utilização de } \\
\text { Equipamentos de Proteção Individual (EPI) pelos } \\
\text { manipuladores, o que variou significativamente } \\
\text { entre as unidades avaliadas. }\end{array}$ \\
\hline $\begin{array}{l}\text { Santos e } \\
\text { Ferreira } \\
(2019)\end{array}$ & $\begin{array}{l}\text { Avaliar a eficácia do treinamento } \\
\text { de manipuladores de alimentos } \\
\text { de uma Unidade de Alimentação } \\
\text { e Nutrição de uma escola do } \\
\text { município de Januária-MG, } \\
\text { visando a redução dos riscos a } \\
\text { saúde para os consumidores. }\end{array}$ & $\begin{array}{l}\text { Aplicação de lista de } \\
\text { verificação de boas práticas } \\
\text { para Unidades de Alimentação } \\
\text { e Nutrição, adaptada da RDC } \\
\text { 275/2002, em uma escola } \\
\text { pública do município, antes e } \\
\text { após treinamento para } \\
\text { manipuladores de alimentos. }\end{array}$ & $\begin{array}{l}\text { A unidade apresentou } 74,5 \% \text { de conformidade } \\
\text { antes, e } 81,8 \% \text { após o treinamento para } \\
\text { manipuladores, passando da classificação } \\
\text { "parcialmente adequada" para "adequado", } \\
\text { conforme a RDC } 275 / 2002 \text {. As principais } \\
\text { inadequações antes do treinamento de } \\
\text { manipuladores estiveram relacionadas aos } \\
\text { processos e procedimentos, especialmente higiene } \\
\text { e higienização das mãos. }\end{array}$ \\
\hline $\begin{array}{l}\text { Brandão } \\
\text { Padilha, } \\
\text { Campos, } \\
\text { Severiano e } \\
\text { Torres (2020) }\end{array}$ & $\begin{array}{l}\text { Avaliar as condições higiênico- } \\
\text { sanitárias de Unidades de } \\
\text { Alimentação e Nutrição de } \\
\text { escolas de Maceió-AL e verificar } \\
\text { a adequação do número de } \\
\text { nutricionistas ao número de } \\
\text { alunos matriculados na rede } \\
\text { pública de ensino municipal. }\end{array}$ & $\begin{array}{l}\text { Aplicação de lista de } \\
\text { verificação de boas práticas } \\
\text { para Unidades de Alimentação } \\
\text { e Nutrição de escolas, proposta } \\
\text { e validada por Stedefeldt } \text { et al } \\
\text { (2013), em } 16 \text { escolas de ensino } \\
\text { infantil do município. }\end{array}$ & $\begin{array}{l}\text { A maioria das unidades apresentou risco sanitário } \\
\text { regular, com } 58 \% \text { de conformidade. O bloco de } \\
\text { recebimento foi o que recebeu melhor avaliação } \\
\text { em todas as unidades e o de equipamentos para } \\
\text { temperatura controlada o de pior avaliação, com } \\
68,75 \% \text { das unidades enquadradas entre risco } \\
\text { sanitário alto e muito alto. }\end{array}$ \\
\hline $\begin{array}{l}\text { Ramos, } \\
\text { Valença, Peter } \\
\text { e Muniz } \\
(2020)\end{array}$ & $\begin{array}{l}\text { Avaliar as boas práticas de } \\
\text { alimentação, através de checklist, } \\
\text { em Unidades de Alimentação e } \\
\text { Nutrição de escolas da rede } \\
\text { municipal da zona rural da } \\
\text { cidade de Pelotas-RS. }\end{array}$ & $\begin{array}{l}\text { Aplicação de lista de } \\
\text { verificação de boas práticas } \\
\text { para Unidades de Alimentação } \\
\text { e Nutrição, adaptada da RDC } \\
275 / 2002 \text {, em } 20 \text { escolas } \\
\text { públicas da zona rural do } \\
\text { município. }\end{array}$ & $\begin{array}{l}\text { A maioria das unidades apresentou } 50 \% \text { de } \\
\text { conformidade, sendo classificadas como } \\
\text { "inadequado" conforme RDC } 275 / 2002 \text {. As } \\
\text { inconformidades estiveram relacionadas à } \\
\text { documentação e registro, manejo de resíduos, } \\
\text { edifícios e instalações e preparação do alimento. }\end{array}$ \\
\hline $\begin{array}{l}\text { Wognski et al. } \\
\text { (2021) }\end{array}$ & $\begin{array}{l}\text { Comparar as cantinas em } \\
\text { diferentes tipos de escolas de } \\
\text { Curitiba-PR, bem como o tipo de } \\
\text { gestão e a presença ou não de } \\
\text { profissionais técnicos no } \\
\text { contexto das Pessoas Jurídicas. }\end{array}$ & $\begin{array}{l}\text { Aplicação de lista de } \\
\text { verificação de boas práticas, } \\
\text { baseada na RDC } 275 / 2002 \text { e na } \\
\text { lista de Stedefeldt } \text { et al. }(2013) \text {, } \\
\text { em } 111 \text { escolas públicas e } \\
\text { privadas de Curitiba. }\end{array}$ & $\begin{array}{l}\text { A maioria das unidades apresentou risco sanitário } \\
\text { regular, com 55,5\% de conformidade. Os pontos } \\
\text { mais insatisfatórios foram armazenamento de } \\
\text { pratos prontos, controle da temperatura dos } \\
\text { alimentos, treinamento de manipuladores e } \\
\text { documentação e registro. }\end{array}$ \\
\hline
\end{tabular}

Legenda: RDC: Resolução da Diretoria Colegiada.

Fonte: Rocha et al. (2021)

\section{Discussão}

A Segurança Alimentar e Nutricional (SAN) está integrada à oferta de alimentos e refeições de qualidade higiênicosanitária (Soares et al., 2018). Assim, a alimentação produzida nas escolas, além de ser adequada sob os aspectos nutricionais e sensoriais, deve ser constituída de alimentos seguros do ponto de vista sanitário (Goulart, 2019).

As BP devem ser adotadas em todas as etapas de produção que possam interferir na segurança do alimento, incluindo instalações, ambiente, produção, manipuladores, fornecedor, equipamentos, abastecimento de água, lixo e esgoto, recebimento, armazenamento, dentre outros (Silva et al., 2016). A avaliação do risco sanitário de Unidades de Alimentação e Nutrição de escolas ou risco de contaminação das refeições ofertadas nesse ambiente, permite identificar de forma mais precisa em que etapa do processo de produção é necessário agir (Soares et al., 2018).

De modo geral, o percentual médio de conformidade das Unidades de Alimentação e Nutrição estudadas foi de $58,9 \%$, sendo classificadas como parcialmente adequadas ou de risco sanitário regular. Um estudo encontrou unidades com condições higiênico-sanitárias adequadas (Mendes et al., 2019), sete observaram unidades com risco sanitário regular ou adequação parcial das boas práticas (Batista \& Vergara, 2017; Soares et al., 2018; Ferro et al., 2018; Rudakoff et al., 2018; 
Santos \& Ferreira, 2019; Brandão et al., 2020; Wognski et al., 2021), e três classificaram as unidades como de risco sanitário alto ou com condições higiênico-sanitárias inadequadas (Fortes et al., 2017; Ribeiro et al., 2018; Ramos et al., 2020). Resultados semelhantes foram anteriormente observados no estudo de Freitas et al. (2017) ao revisar a literatura acerca do sistema de segurança dos alimentos em escolas de educação infantil, na qual os autores destacaram que as condições higiênicosanitárias das áreas de produção dos alimentos nessas unidades estavam fora dos padrões estabelecidos na legislação, o que implica vários fatores de risco para a saúde das crianças atendidas.

Nas Unidades de Alimentação e Nutrição classificadas como de risco sanitário regular, as inadequações foram observadas nos blocos edifício e instalações, equipamentos, higienização ambiental e processos e procedimentos (Batista \& Vergara, 2017; Soares et al., 2018; Ferro et al., 2018; Rudakoff et al., 2018; Santos \& Ferreira, 2019; Brandão et al., 2020; Wognski et al., 2021). As unidades com risco sanitário alto, embora os índices de conformidade tenham sido inadequados em todos os blocos avaliados, os mais críticos estiveram relacionados ao recebimento, manipuladores e processos e procedimentos (Fortes et al., 2017; Ribeiro et al., 2018; Ramos et al., 2020).

A maioria dos estudos identificou pelo menos uma inadequação associada aos edifícios e instalações, como: presença de animais, acúmulo de lixo ou entulhos e esgoto aberto nas imediações das unidades; paredes e/ou tetos mal conservados, com infiltrações, descascamento ou rachaduras ou ainda teto sem forro; portas e janelas não ajustadas aos batentes, janelas sem telas milimétricas, permitindo a entrada de vetores e pragas; ausência ou inadequações nos ralos da cozinha; ventilação deficiente e utilização de ventiladores na cozinha; luminárias sem proteção contra quedas acidentais na área de preparo; ausência de lavatórios e de sanitários e vestiários de uso exclusivo dos manipuladores de alimentos (Batista \& Vergara, 2017; Fortes et al., 2017; Soares et al., 2018; Ferro et al., 2018; Rudakoff et al., 2018; Ribeiro et al., 2018; Brandão et al., 2020; Ramos et al., 2020; Santos \& Ferreira, 2019). No estudo de Mendes et al. (2019), único que encontrou condições higiênico-sanitárias adequadas nas unidades estudadas, houve inadequações no bloco de edifícios e instalações, com a ausência de lixeiras com acionamento por pedal, de portas com acionamento automático e de proteção contra insetos e roedores.

As edificações exercem influência positiva ou negativa sobre a segurança dos alimentos produzidos nas Unidades de Alimentação e Nutrição (Gomes, Campos \& Monego, 2012). Rudakoff et al. (2018) observaram correlação positiva e moderada entre os escores obtidos de higienização ambiental e edificações, demonstrando que a estrutura física e o ambiente desses locais, quando inadequados, interferem de modo negativo na segurança dos alimentos neles manipulados. Segundo esses autores, índices altos de inconformidades nos edifícios e instalações de Unidades de Alimentação e Nutrição de escolas podem ser decorrentes da adaptação destas aos espaços físicos disponíveis no ambiente escolar.

Quanto à higienização ambiental, Kochansky et al. (2009) destacam que medidas adequadas de higienização de equipamentos, móveis e utensílios podem promover maior segurança microbiológica nos estabelecimentos de alimentação coletiva. Entretanto, os estudos identificaram a presença de pragas urbanas e falhas na higienização de utensílios, esponjas, panos e equipamentos, como o uso de panos de louça não descartáveis para higienizar balcões, mesas e prateleiras e o armazenamento de utensílios sobre balcões ou em prateleiras abertas, expondo-os a insetos e poeira, aumentando o risco de contaminação (Fortes et al., 2017; Soares et al., 2018). No estudo de Wognski et al. (2021), embora a higienização ambiental tenha sido o bloco com maior adequação, o armazenamento e exposição de pratos prontos para o consumo teve o pior índice de conformidade $(22,1 \%)$, reforçando a essencialidade da adoção das boas práticas em todas as etapas de produção do alimento.

No que se refere ao bloco de equipamentos para temperatura controlada, os estudos destacaram a ausência ou insuficiência de geladeiras e câmaras frias e de termômetro para aferição da temperatura dos alimentos, bem como de balcão quente (Batista \& Vergara, 2017; Fortes et al., 2017; Brandão et al., 2020). De acordo com a RDC n $216 / 2004$ da Anvisa, deve ocorrer o monitoramento e registro de temperatura das matérias-primas, ingredientes e equipamentos durante a recepção e armazenamento. Além disso, é necessário monitorar a temperatura dos alimentos prontos para consumo de modo a mantê-las 
em valores $\geq 60^{\circ} \mathrm{C}$ para alimentos quentes e $\leq 5^{\circ} \mathrm{C}$ para alimentos frios, visando minimizar o risco de ocorrência de doenças transmitidas por alimentos (Anvisa, 2004).

No recebimento de matérias-primas, materiais e insumos deve ocorrer uma análise qualitativa e quantitativa dos gêneros, na qual é essencial a verificação das condições da embalagem e do prazo de validade visando contribuir para a manutenção das condições higiênico-sanitárias e nutricionais dos alimentos manipulados (Celestino et al., 2017). Apesar desse bloco não ter sido o de pior adequação na maioria dos estudos, Fortes et al. (2017) verificaram que por não haver funcionários responsáveis pelo recebimento das matérias-primas nas Unidades de Alimentação e Nutrição avaliadas, as condições das matérias-primas, das embalagens, data de validade, rotulagem e qualidade das mesmas não eram observadas pelos manipuladores no momento da entrega.

Quanto aos manipuladores de alimentos, não são incomuns inadequações relacionadas principalmente ao uso de uniformes e de adornos, como é destacado por Ribeiro et al. (2018), ao observarem que os manipuladores de todas as unidades analisadas em seu estudo não utilizavam o uniforme completo, sendo que alguns utilizavam bermudas, regatas, saias e chinelos, bem como brincos, alianças e fones de ouvido. De modo semelhante, Ferro et al. (2018) encontraram apenas 12,5\% dos manipuladores das unidades de Tocantins devidamente uniformizados. Sabendo que os manipuladores representam fator essencial na segurança da produção de alimentos é importante que realizem as atividades de acordo com as recomendações da legislação sanitária: manter as unhas aparadas e sem esmalte; manter as mãos sempre limpas; utilizar uniformes limpos, touca, e sapatos fechados; e não utilizar barba e/ou bigode ou qualquer tipo de adorno como brincos, pulseiras, anéis etc. (Anvisa, 2004). A utilização de adornos e a baixa adesão ao uso de uniformes podem estar associadas a fatores como falta de capacitação, materiais e estrutura física para a adequada higienização das mãos e utilização dos Equipamentos de Proteção Individual (EPI), promovendo risco para a saúde dos estudantes (Rezer, 2015). A capacitação dos manipuladores de Unidade de Alimentação e Nutrição de uma escola pública de Januária-MG foi capaz de aumentar o percentual de conformidade às boas práticas de 74,5\% para 81,5\%, melhorando as condições higiênico-sanitárias da unidade (Santos \& Ferreira, 2019).

No item de processos e procedimentos foram observadas falhas na higienização e higiene das mãos e no descongelamento de alimentos bem como ausência de Manual de Boas Práticas (MBP), Procedimentos Operacionais Padronizados (POP) e outros registros (Santos \& Ferreira, 2019; Ramos et al., 2020; Rudakoff et al., 2018; Wognski et al., 2021). Rudakoff et al. (2018) não identificaram MBP, POP e nem registro do controle de temperatura e características do recebimento, da temperatura de cocção e da distribuição em nenhuma das 30 unidades de alimentação em escolas de São Luís MA. A ausência de documentação e registro, referentes ao MBP e POP, também foi observada por Ramos et al. (2020) na maioria das 20 escolas avaliadas, na zona rural de Pelotas-RS. Santos \& Ferreira (2019) ressaltaram que a ausência de lavatório exclusivo na área de manipulação, de sabonete líquido inodoro antisséptico e papel toalha bem como de informes sobre o procedimento correto de lavagem, podem contribuir para o baixo percentual de adequação do procedimentos de higiene e higienização das mãos.

A inexistência do MBP e de POP em unidades de alimentação contrariam a RDC no 216/2004 da Anvisa, a qual estabelece a existência obrigatória destes documentos em estabelecimentos produtores de refeições com vistas a aplicar as normas na unidade e garantir a segurança dos alimentos ofertados (Anvisa, 2004). Considerando que os documentos supracitados contêm as atividades realizadas pela Unidade de Alimentação e Nutrição para garantir a produção de refeições saudáveis e seguras, a presença dos mesmos pode representar uma garantia da execução das boas práticas na instituição (Rudakoff et al., 2018). É também estabelecido na RDC nº 216/2004 que a área de manipulação de alimentos deve conter lavatórios exclusivos para a higiene das mãos, suficientes e em posições estratégicas em relação ao fluxo de preparo dos alimentos, sendo munidos de sabonete líquido inodoro antisséptico ou sabonete líquido inodoro e produto antisséptico, toalhas de papel não reciclado ou outro sistema higiênico e seguro de secagem das mãos e coletor de papel acionado por pedal 
(Anvisa, 2004). Segundo Moreira, Peder e Silva (2016), a higienização adequada das mãos pode reduzir o risco de contaminação dos alimentos por Staphylococcus aureus, um dos principais microrganismos patogênicos causadores de intoxicação alimentar.

Estudos sugerem que o nutricionista desempenha papel importante nas condições higiênico-sanitárias de Unidades de Alimentação e Nutrição de escolas, uma vez que a presença ou atuação desse profissional nesses estabelecimentos contribui para adoção de práticas de higiene adequadas, o que pode melhorar os aspectos sanitários das unidades. Isso foi demonstrado por Santos e Ferreira (2019) que verificaram melhora significativa de conformidade às boas práticas em uma cantina escolar após nutricionistas realizarem treinamento com os manipuladores de alimentos da unidade. Wognski et al. (2021) observaram que as escolas que possuíam nutricionista como responsável técnico pela unidade de alimentação obtiveram melhores resultados de conformidade às boas práticas na maioria das categorias avaliadas quando comparadas às unidades sem a responsabilidade técnica desse profissional.

\section{Conclusão}

Diante do exposto, observa-se que a maioria das Unidades de Alimentação e Nutrição de escolas brasileiras avaliadas apresentaram condições higiênico-sanitárias parcialmente adequadas em relação às boas práticas, sendo classificadas como de risco sanitário regular. As não conformidades estiveram presentes nos diferentes blocos avaliados, variando entre os estudos, sendo frequente a verificação de falhas nos edifícios e instalações das unidades, que associadas a outros fatores ou não, influenciam negativamente a segurança sanitária dos alimentos produzidos, aumentando o risco de contaminação dos alimentos e de ocorrência de doenças transmitidas por alimentos.

Os resultados desse estudo demonstram a necessidade de maior atenção dos órgãos de controle sanitário para com as Unidades de Alimentação e Nutrição de escolas no Brasil, uma vez que a alimentação ofertada nesses ambientes deve ser de qualidade nutricional e sanitária, de modo a contribuir para a saúde e rendimento acadêmico dos estudantes. Evidencia-se a importância do nutricionista como responsável técnico de cantinas escolares, pois a atuação deste profissional contribui para adoção e manutenção de práticas de higiene e manipulação de alimentos em conformidade com as recomendações da legislação sanitária.

\section{Referências}

Batista, A. S. S., \& Vergara, C. M. A. C. (2017). Verificação das boas práticas e do risco sanitário em unidades de alimentação e nutrição escolares do município de Maracanaú, CE. Higiene Alimentar, 31(268/269), 51-55. https://pesquisa.bvsalud.org/portal/resource/pt/biblio-837464

Brandão, T. B. C., Padilha, B. M., Campos, E. L. L., Severiano, G. M. L., \& Torres, J. C. L. (2020). Adequação numérica de nutricionistas e condições higiênico-sanitárias de Unidades de Alimentação e Nutrição Escolares de Maceió, Alagoas. Segurança Alimentar e Nutricional, 27, e020026, 1-8. $10.20396 /$ san.v27i0.8659559

Agência Nacional de Vigilância Sanitária (Anvisa). (2004). Resolução RDC $n^{\circ}$ 216, de 15 de setembro de 2004. Dispõe sobre Regulamento Técnico de Boas Práticas para Serviços de Alimentação. Diário Oficial da União. Brasília: Anvisa. https://bvsms.saude.gov.br/bvs/saudelegis/anvisa/2004/res021 6_15_09_2004.html

Agencia Nacional de Vigilância Sanitária (Anvisa). (2002). Resolução RDC $n^{\circ} 275$, de 21 de outubro de 2002. Dispõe sobre o Regulamento Técnico de Procedimentos Operacionais Padronizados aplicados aos Estabelecimentos Produtores/Industrializadores de Alimentos e a Lista de Verificação das Boas Práticas de Fabricação em Estabelecimentos Produtores/Industrializadores de Alimentos. Diário Oficial da União. Anvisa. http://portal.anvisa.gov.br/documents/10181/2718376/RDC_275_2002_COMP.pdf/fce9dac0-ae57-4de2-8cf9-e286a383f254

Celestino, A. F., Jr., Matos, E. C. O., Filocreão, B. L., Silva, C. C., Soares, M. I. S., \& Costa, R. C. L. (2017). Riscos infecciosos no ambiente escolar: relato de experiência com escolares através de metodologia ativa. Saúde \& Transformação Social, 8(2), 128-134. http://incubadora.periodicos.ufsc.br/index.php/saudeetransformacao/article/view/3661/4992

Conselho Federal de Nutricionistas (CFN). (2018). Resolução $C F N n^{\circ}$ 600, de 25 de fevereiro de 2018. Dispõe sobre a definição das áreas de atuação do nutricionista e suas atribuições, indica parâmetros numéricos mínimos de referência, por área de atuação, para a efetividade dos serviços prestados à sociedade e dá outras providências. Brasília: CFN. https://www.cfn.org.br/wp-content/uploads/resolucoes/Res_600_2018.htm. 
Ferreira, C. A., Lima, V. S, \& Aguiar, L. P. (2020). Condições higiênicos sanitárias dos serviços de alimentação no Brasil: uma revisão integrativa. Research, Society and Development, 9(10), e499108156. 10.33448/rsd-v9i10.8156

Ferrari, A. M., \& Fonseca, R. V. (2019). Conhecimento de consumidores a respeito de doenças transmitidas por alimentos. UNESC em Revista, 3(1), 1-12. http://revista.unesc.br/ojs/index.php/revistaunesc/article/view/56

Ferro, L. L., Fialho, C. J., Pires, C. R. F., Teles, N. B., \& Santos, V. F. (2018). Condições higiênico sanitárias de Unidades de Alimentação e Nutrição de escolas públicas do estado do Tocantins. Segurança Alimentar e Nutricional, 25(2),118-130. 10.20396/san.v25i2.8651815

Fortes, K. S. B., Brasil, C. C. B., Silva, J. P., Pontes, B. D., \& Graupe, M. L. (2017). Condições higiênico-sanitárias de unidades de alimentação e nutrição de escolas de educação infantil de Palmeira das Missões - RS. Vigilância Sanitária em Debate, 5(3), 37-43. https://visaemdebate.incqs.fiocruz.br/index.php/visaemdebate/article/view/959

Freitas, A. A. M., Cunha, F. M. F., Machado, F. M., Silva, N. C., \& Raghiante, F. (2017). Avaliação da segurança dos alimentos nas escolas infantis. Nutrição Brasil, 16(4), 243-249. 10.33233/nb.v16i4.1277

Gomes, N. A. A. A., Campos, M. R. H., \& Monego, E. T. (2012). Aspectos higiênico-sanitários no processo produtivo dos alimentos em escolas públicas do Estado de Goiás, Brasil. Revista de Nutrição, 25(4), 473-485. 10.1590/S1415-52732012000400005

Goulart, J. L. P. (2019). Avaliação do risco higiênico-sanitário a partir da aplicação da ferramenta de boas práticas na alimentação escolar nas unidades de ensino de Ribeirão Preto, São Paulo (Dissertação de mestrado). Universidade de São Paulo-USP, Ribeirão Preto, São Paulo, Brasil. https://teses.usp.br/teses/disponiveis/17/17139/tde-01062020-085343/pt-br.php

Kochansky, S., Pierozan, M. K., Mossi, A. J., Treichel, H., Cansian, R. L., Ghisleni, C. P., \& Toniazzo, G. (2009). Avaliação das condições microbiológicas de uma unidade de alimentação e nutrição. Revista Alimentos e Nutrição, v.20(4), 663-668. http://serv-bib.fcfar.unesp.br/seer/index.php/ alimentos/article/viewFile/1264/873

Martins, M., \& Spinelli, M. G. N. (2020). Avaliação de rendimento, custo e aceitação de carnes bovinas e suínas utilizadas em unidades de alimentação e nutrição. Revista Saber Científico, 9(1), 45-53. 10.22614/resc-v9-n1-1308

Mendes, T. I. L., Monteiro, M. L. S., Carvalho, L. M. F., \& Bezerra, K. C. B. (2019). Condições higiênicas e sanitárias de unidades de alimentação e nutrição de escolas em tempo integral. Revista Eletrônica Acervo Saúde, 31(Supl.), e1150. 10.25248/reas.e1150.2019

Moreira, B. G., Peder, L. D., \& Silva, C. M. (2016). Prevalência de Staphylococcus aureus nos vestíbulos nasais e mãos de manipuladores de alimentos em um hospital do oeste do Paraná, Brasil. Revista UNINGA Review, 28(1), 51-55. http://revista.uninga.br/index.php/uningareviews/article/view/1846

Oliveira, A. G. D., Oliveira, C. F., Mallet, A. C. T., \& Souza, H. L. S. (2020). O nutricionista como promotor de qualidade e lucratividade em unidades de alimentação e nutrição. Revista Episteme Transversalis, 11(3), 120-140. http://revista.ugb.edu.br/ojs302/index.php/episteme/article/view/2182/1338

Ramos, C. I., Valença, M. S., Peter, N. B., \& Muniz, L. C. (2020). Avaliação das boas práticas em Unidades de Alimentação Escolar da zona rural do município de Pelotas, RS. Semina: Ciências Biológicas e da Saúde, 41(1), 67-74. 10.5433/1679-0367.2020v41n1p67

Rezer, D. S. (2015). Alimentação escolar: Recursos humanos e equipamentos em escolas públicas de Porto Alegre/RS (Trabalho de conclusão de curso). Universidade Federal do Rio Grande do Sul-UFRGS, Porto Alegre, Rio Grande do Sul, Brasil. https://lume.ufrgs.br/handle/10183/143718.

Ribeiro, J. A., Damaceno, K. J. L., Moura, K. D. L., Salvador, A. A., Rossetti, F. X., Tamasia, G. A., Bello, S. R. B., \& Vicentini, M. S. (2018). Análise das condições higiênico sanitárias das unidades de alimentação e nutrição das escolas de um município no Vale do Ribeira, SP. Research, Society and Development, 7(8), e478327. 10.17648/rsd-v7i8.327

Rudakoff, L. C. S., Mouchreck, A. N., Frota, M. T. B. A., \& Bastos, L. S. (2018). Qualidade e Segurança Alimentar em unidades de alimentação e nutrição escolares da rede municipal de educação de São Luís, Maranhão. Vigilância Sanitária em Debate, 6(3), 46-53. doi: 10.22239/2317-269x.01094

Santos, J. M., \& Ferreira, L. C. (2019). Condições higiênico-sanitárias em uma escola pública do município de Januária-MG antes e após o treinamento dos manipuladores de alimentos. Caderno de Ciências Agrárias, 11, 1-7. 10.35699/2447-6218.2019.12285

Silva, L. M., Brasil, C. C. B., Marques, A. C., Silveira, J. T., Moura, F. A., Martini, C. S., \& Schiavo, M. B. (2016). Avaliação das condições higiênicas de escolas de ensino infantil e fundamental por meio da aplicação de listas de verificação. Segurança Alimentar e Nutricional, 23(1), 837-848. 10.20396/san.v23i1.8642256

Soares, D. S. B., Henriques, P., Ferreira, D. M., Dias, P. C., Pereira, S., \& Barbosa, R. M. S. (2018). Boas Práticas em Unidades de Alimentação e Nutrição Escolares de um município do estado do Rio de Janeiro - Brasil. Ciência \& Saúde Coletiva, 23(12), 4077-4083. 10.1590/1413-812320182312.23992016

Sousa, A. A., Paz, F. A. M., Carvalho, L. M. F., Bezerra, K. C. B., \& Landim, L. A. S. R. (2020). Condições higiênico-sanitárias em unidades produtoras de refeições: uma revisão. Research, Society and Development, 9(11), e46891110017. 10.33448/rsd-v9i11.10017

Stedefeldt, E., Cunha, D. T., Silva Júnior, E. A., Silva, S. M., \& Oliveira, A. B. A. (2013). Instrumento de avaliação das Boas Práticas em Unidades de Alimentação e Nutrição Escolar: da concepção à validação. Ciência \& Saúde Coletiva, 18(4), 947-953. 10.1590/S1413-81232013000400006

Wognski, A. C. P., Choma, C., Gava, G. R., Ferreira, B. G. C. S., Vieira, L. P., Oliveira, E. C., Santos, V. C., Mezzomo, T. R., \& Stangarlin-Fiori, L. (2021). Good hygiene practices in school canteens: evaluation between types of schools and administration as well as presence of technical professional. Brazilian Journal of Food Technology, 24, e2019257. 10.1590/1981-6723.25719 